\title{
Measuring the passive cuticular membrane permeability of potassium with a parallel artificial membrane permeability assay and the implications for foliar nutrient formulations
}

\author{
Qingwen $\mathrm{He}^{*}$ (D) and Taha Rezai
}

\begin{abstract}
A modified PAMPA (parallel artificial membrane permeability assay) is proposed for evaluating the passive cuticular membrane permeability of potassium in several foliar nutrient formulations. The modified PAMPA can measure the passive permeability of ionic nutrients under fully hydrated conditions through an artificial membrane designed to more closely resemble a plant cuticle, rather than the traditional phospholipid animal model. Foliar nutrient formulations, which in some cases contain a complex organic matter component, may be evaluated with the modified PAMPA in order to develop better structure activity relationships that can help guide rational formulation development. In addition, mechanistic insights may also be uncovered with the simplified system.
\end{abstract}

Keywords: Passive membrane permeability, Potassium, PAMPA, Foliar nutrient, Formulations

\begin{abstract}
Introduction
Potassium is a critical plant macronutrient. It functions as an enzyme cofactor and is the principal cation in establishing cell turgor and maintaining cell electroneutrality [1]. Although most potassium uptake is through fine root hairs, foliar applications are often used to supplement soil fertilization. The waxy cuticle is known to be the main barrier to foliar penetration of water-soluble ions like potassium [1]. Upon foliar application, where external leaf potassium levels will be high, the water-soluble ion will cross the apoplast (the cuticle and cell wall of the leaf cell) passively. At high concentrations potassium will then passively enter the cellular cytosol and is only actively transported into the plant cell when extracellular potassium levels are very low [2].
\end{abstract}

${ }^{*}$ Correspondence: karen.he@actagro.com

Actagro Technology and Innovation Center, 4516 N. Howard Ave., Gate 1, Kerman, CA 93630, USA
Many factors influence the foliar penetration of ions including spray droplet formation, droplet retention, wetting, spreading, hydration and permeation as well as others [3]. Established laboratory assays like simulation of foliar uptake (SOFU) take into consideration environmental, spray-dependent factors and cuticular penetration of the active ingredient [4]. In fact, it has been previously shown with SOFU that potassium cuticle penetration of various salts is a purely physical process that follows first-order rate kinetics and is strongly influenced by the point of deliquescence (POD) of the salt used and the relative humidity [5]. Another study on calcium cuticle penetration using SOFU also concluded that keeping salts wet on the leaf is the most important factor in foliar nutrition, since this allows for ion penetration through the aqueous pores in the cuticle via the hydrophilic pathway [6]. In addition, high relative humidity causes the water content of the cuticle to swell, resulting in an increase in the number and radius of aqueous 
pores which in turn facilitates the transport of ionic compounds [7]. As a result of this and other work, the only adjuvants recommended for improving penetration of ions through the cuticular hydrophilic pathway are wetting agents that help keep applied solutions wet longer and contribute to swelling the cuticle.

More recently, an in silico porous diffusion model has been developed to simulate diffusion of hydrophilic ions through a plant cuticle. Interestingly, the major factor influencing uptake in the model was found to be cuticle structure, including tortuosity and density of the aqueous pores. Relative humidity and binding of ions to the surface of cuticles, although still rated high after sensitivity analysis, were less influential [8]. An ion-pairing mechanism has also been proposed as a method to increase the permeability of very polar positively charged cationic drugs through membranes. In ion-pairing, the addition of lipophilic anions allows for the formation of neutral ion pairs that together can more easily cross a hydrophobic barrier [9-11]. These results suggest that looking more closely at the process of passive permeability of ions across the plant cuticle may reveal additional mechanistic insights that can assist rational formulation development. In order to do that, passive cuticular membrane permeability must first be uncoupled from all the other factors that influence foliar nutrient uptake through the use of a simple experimental setup. This would be particularly helpful when evaluating formulations which combine both nutrients and complex organic mixtures that have a range of physical as well as biological effects that are difficult to measure.

In this work we focus on measuring the passive cuticular membrane permeability of potassium in proprietary and commodity potassium formulations using PAMPA (parallel artificial membrane permeability assay). PAMPA was first introduced in 1998 for evaluating the passive membrane permeability of pharmaceuticals in the gastrointestinal tract [12]. More recently PAMPA has also been used to evaluate the passive membrane permeability of crop protection compounds [13], but to our knowledge has not been used previously to simulate nutrient transport across a leaf cuticle. The PAMPA setup consists of an artificial lipid barrier resting on a filter support in a 96-well plate format. The ability of an analyte to diffuse from the donor compartment, through the membrane, into a Teflon-coated acceptor well is measured. PAMPA has a simple design and the 96-well plate format makes it amenable to automation and high-throughput analysis. Evaluating foliar nutrient formulations with PAMPA is of interest, since the assay can be used to selectively assess the passive cuticular membrane permeability of a nutrient, without other confounding effects (active transport, biological, environmental and spray application dependent). Most importantly for our purposes, in PAMPA a lipid is sandwiched between two liquid compartments (Fig. 1), so that passive cuticular membrane permeability is purely assessed under fully hydrated conditions where relative humidity and the POD of salts are no longer influencing variables. In addition, under the conditions of PAMPA, the cuticular membrane will be completely swelled with water.

\section{Methods/experimental}

Parallel artificial membrane permeability assay

PAMPA Donor (MAIPNTR10) and Acceptor plates (MSSACCEPTOR) were purchased from Millipore. Phosphatidylcholine is typically used as the lipid in PAMPA when evaluating drug permeability for animals. In this study we instead chose to use a mixture of stearic acid (98\%, Alfa Aesar) $n$-Triacontane (99\%, BTC)

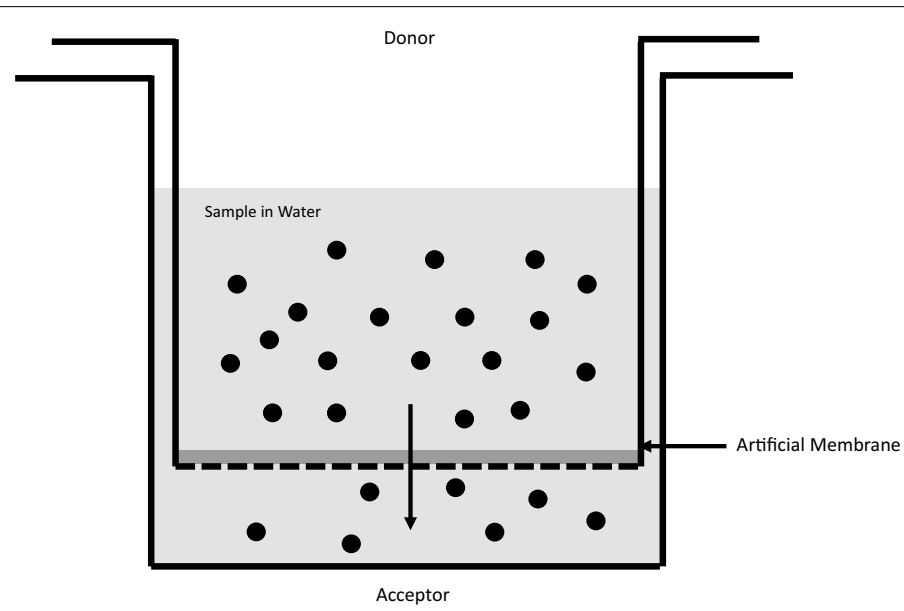

Fig. 1 Simple diagram of a single PAMPA well with analyte moving from donor to acceptor well 
and hexadecane (99\%, Alfa Aesar). Stearic acid is a fatty acid that is similar to the monomers that make up the waxy cuticle of plants [14] and the addition of hydrophobic alkanes provide water repellency [15]. The PAMPA lipid solution was prepared at a concentration of $0.25 \%$ $(\mathrm{w} / \mathrm{v}) n$-Triacontane, $0.25 \%(\mathrm{w} / \mathrm{v})$ stearic acid and $11 \%$ $(\mathrm{v} / \mathrm{v})$ hexadecane in hexane. The lipid solution was then sonicated before use to ensure a homogenous suspension. PAMPA donor solutions were prepared by diluting each sample to $25 \mathrm{mM}$ potassium in DI Water. Activator 90 (Loveland Products, Loveland, CO) was also added to donor solutions at a concentration of $0.05 \%(\mathrm{v} / \mathrm{v})$ as a non-ionic wetting agent. The PAMPA acceptor solutions consisted of an aqueous citric acid buffer at $10 \mathrm{mM}$ with a $\mathrm{pH}$ of 6 and had an electrical conductivity that was similar to each donor solution in order to maintain electroneutrality.

To prepare the plates, $5 \mu \mathrm{L}$ of a lipid solution was carefully added to each donor well then dried for $1 \mathrm{~h}$. Next, $100 \mu \mathrm{L}$ of donor solution was added to each donor well and $290 \mu \mathrm{L}$ of acceptor solution was added to each acceptor well. The PAMPA donor plate was then carefully stacked on top of the acceptor plate and the PAMPA sandwich was allowed to incubate at high humidity conditions, in order to minimize water loss, for $2 \mathrm{~h}$. Each sample was run on PAMPA with 4 replicates. At the end of the incubation period acceptor well potassium concentrations were quantified by ICP-OES at a certified thirdparty laboratory. For Lucifer Yellow, UV-Vis absorbance at $280 \mathrm{~nm}$ was used for quantitation of acceptor wells by the serial dilution method.

\section{Log $\mathrm{P}_{\mathrm{e}}$ and \%transmittance}

PAMPA directly measures the effective permeability (log $\mathrm{Pe}$ ) of analytes with the following formula [12]: proprietary complex organic matter component (17.5\% Total Carbon). In addition to Katalyst $\mathrm{FS}^{\circledR}$, potassium chloride (99.0-100.5\%, Alfa Aesar) and potassium acetate $(\geq 99.0 \%, \mathrm{BDH})$ solutions were prepared for use in PAMPA, all at $25 \mathrm{mM}$ starting potassium concentrations. Lucifer Yellow (>95\%, Biotium) donor solutions were prepared at a concentration of $0.16 \mathrm{mg} / \mathrm{mL}$, as previously described [17].

\section{Results and discussion}

In order to validate the modified PAMPA several steps were taken. First, the permeability of potassium was evaluated over time. As can be seen in Fig. 2, the \% $\mathrm{T}$ vs time graph for Katalyst $\mathrm{FS}^{\circledR}$ displays a first order hyperbolic curve that is typical of passive membrane permeability.

The mass balance of potassium for the system was also determined in order to ensure that all potassium in the system was accounted for. This was done by quantifying potassium in the donor and acceptor wells at multiple time points during the Katalyst FS ${ }^{\circledR}$ incubation. Table 1 shows that the total potassium in the system at selected points in the Katalyst $\mathrm{FS}^{\circledR}$ assay from Fig. 2 are close to initial values and almost all the potassium is accounted for. The data in Table 1 also tells us that there is a negligible amount of potassium associated with the membrane or adsorbed to the surface of the PAMPA apparatus.

Finally, in order to test whether or not the membrane applied remained intact during incubation, Lucifer Yellow was run in the modified PAMPA. It is well known that Lucifer Yellow has low passive membrane permeability and is commonly used to test the integrity of artificial membranes [17]. Results show that after $2 \mathrm{~h}$ of incubation in the modified PAMPA, only $0.15 \%$ of the donor concentration ended up in the acceptor well. This is comparable to results previously found for Lucifer Yellow on

$$
\log \mathrm{Pe}=\log \{\mathrm{C} \cdot-\ln (1-[\text { analyte]acceptor } /[\text { analyte]equilibrium })\}
$$

where $\mathrm{C}=(\mathrm{V}$ Donor well $\cdot \mathrm{V}$ Acceptor well/(V Donor well $\cdot V$ Acceptor well) Area plate $\cdot$ time).

$\log \mathrm{Pe}$ is a rate with units of $\mathrm{cm} / \mathrm{s}$. Alternatively, permeability can also be followed more directly as the percentage of compound permeated into the acceptor compartment divided by the concentration at equilibrium after a given time or $\% \mathrm{~T}$ [16]. The formula for $\% \mathrm{~T}$ is:

$$
\% \mathrm{~T}=100 \times([\text { analyte }] \text { acceptor } /[\text { analyte]equilibrium })
$$

\section{Samples}

A commercially available product called Katalyst $\mathrm{FS}^{\circledR}$ was obtained from Actagro LLC (Fresno, CA). Katalyst $\mathrm{FS}^{\circledR}$ contains $8.7 \mathrm{M}$ Potassium $\left(30 \% \mathrm{~K}_{2} \mathrm{O}\right)$ as well as a intact PAMPA membranes, where less than $0.2 \%$ of the original donor concentration ended up in the acceptor well after $2 \mathrm{~h}$ [17]. As a result of this and the data presented in both Fig. 2 and Table 1, we have confidence that the modified PAMPA behaves as expected and is a good in vitro system.

Results of potassium permeability for Katalyst FS ${ }^{\circledR}$, potassium chloride and potassium acetate are shown in Table 2.

Katalyst $\mathrm{FS}^{\circledR}$ can be seen to have a potassium permeability that is more than 2 times greater than either potassium chloride or potassium acetate, as shown by $\% \mathrm{~T}$. Although absolute permeabilities may be of little direct field use due to the in-vitro nature of the PAMPA 
\%T vs Time for Katalyst FS ${ }^{\circledR}$

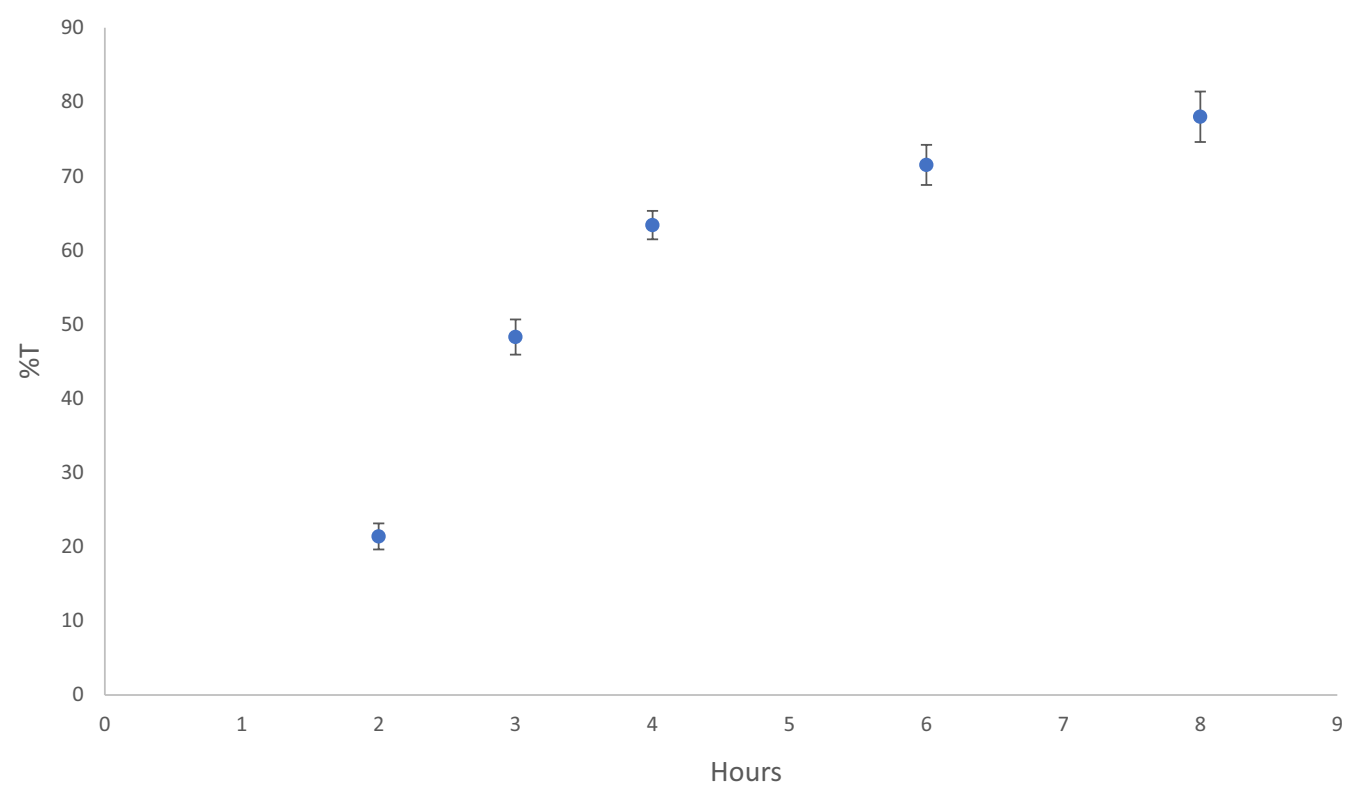

Fig. $2 \%$ T vs time for Katalyst FS ${ }^{\circledR}$

Table 1 Mass balance-percent of initial potassium in combined donor and acceptor solutions at various incubation times

\begin{tabular}{ll}
\hline Time point (h) & $\begin{array}{l}\text { \% initial potassium } \\
\text { in donor and acceptor } \\
\text { solutions }\end{array}$ \\
\hline 0 & 100 \\
2 & 97 \\
3 & 98 \\
4 & 99 \\
\hline
\end{tabular}

Table 2 Modified PAMPA potassium permeability results

\begin{tabular}{|c|c|c|}
\hline Sample & $\log P e^{* a}$ & $\% \mathrm{~T}^{*}$ \\
\hline Katalyst FS ${ }^{\circledR}$ & $-4.99 \pm 0.04 \mathrm{~A}$ & $21.38 \pm 1.76 \mathrm{~A}$ \\
\hline Potassium Chloride & $-5.43 \pm 0.08 B$ & $8.39 \pm 1.46 \mathrm{~B}$ \\
\hline Potassium Acetate & $-5.39 \pm 0.11 \mathrm{~B}$ & $9.38 \pm 2.32 B$ \\
\hline
\end{tabular}

* Means with the same letter are not significantly different $(p<0.05)$ according to Fisher's LSD Method

a Log Pe was converted to Pe in order to ensure a normal distribution, prior to ANOVA and grouping with Fisher's LSD method

assay, relative permeabilities may provide a quick relative assessment of passive cuticular permeability under fully hydrated conditions.
Natural organic matter is known to increase permeability of either biological or artificial membranes [18, 19]. The presence of organic matter outside the membrane may induce the formation of aqueous pores, which are known to allow for hydrophilic ionic transport [20]. Alternatively, or in parallel, polyanionic molecules in the complex organic matter found in Katalyst $\mathrm{FS}^{\circledR}$ may allow for the formation of ion-pairing with potassium. These neutral species may then cross the membrane more efficiently with the help of the lipophilic Katalyst $\mathrm{FS}^{\circledR}$ carrier anions, by means of the traditional solubility-diffusion model of permeability [21]. In order to investigate the feasibility of the ion-pairing hypothesis, UV absorbance was used to track the movement of organic anions from donor to acceptor wells for Katalyst FS ${ }^{\circledR}$. Specifically, a wavelength of $237 \mathrm{~nm}$ that is associated with aromatic molecules known to be present in Katalyst $\mathrm{FS}^{\circledR}$ was used. After subtracting background contributions from the buffer in the acceptor well, an average absorbance of $0.036 \pm 0.002$ was measured. This result demonstrates that organic molecules from Katalyst $\mathrm{FS}^{\circledR}$ are crossing the artificial membrane, a prerequisite for ion-pairing.

\section{Conclusions}

Established laboratory assays for foliar nutrient uptake like SOFU, require the use of radiolabeled analytes due to the small volumes and background interference from nutrients already present in the background matrix. However, the use of radiolabeled analytes creates a 
barrier for most laboratories. The modified PAMPA assay proposed here, allows for a reproducible and highthroughput alternative that is easily accessible and does not require the use of hazardous materials. Although the PAMPA assay proposed only measures passive cuticular membrane permeability of nutrients under fully hydrated in vitro conditions, we believe it may allow for better mechanistic understandings and the development of structure-activity relationships that have the potential to drive formulation improvements. In the case of Katalyst $\mathrm{FS}^{\circledR}$, results suggest that the ion-pairing mechanism is possible and may play a role in the enhanced potassium cuticular permeability of the product.

\section{Abbreviations}

PAMPA: parallel artificial membrane permeability assay; SOFU: simulation of foliar uptake; POD: point of deliquescence; Pe: effective permeability; V: volume; \%T: the percentage of compound permeated into the acceptor compartment divided by the concentration at equilibrium after a given time.

\section{Acknowledgements}

We would like to thank our colleague Dr. Chenping Xu for providing guidance on the design of the artificial cuticular membrane and helpful edits to the paper. We would also like to thank Dr. John Vaughn for insightful discussions related to the ion-pairing experiment.

\section{Authors' contributions}

$\mathrm{QH}$ conducted all of the lab work and optimized the conditions for the modified method proposed in this manuscript. TR proposed the original project, provided guidance during experimental work and wrote the initial draft of the manuscript. Both authors read and approved the final manuscript.

\section{Funding}

Actagro, a subsidiary of Nutrien, provided the funding for this work.

\section{Availability of data and materials}

All relevant data associated with this study are included in this published article. Any other detailed info can be made available by the corresponding author upon reasonable request.

\section{Ethics approval and consent to participate}

No ethics approval and consent to participate needed for any aspect of this study.

\section{Consent for publication}

Authors have reviewed and approved the manuscript for submission.

\section{Competing interests}

Authors are employees of Actagro, a subsidiary of Nutrien.

Received: 17 June 2019 Accepted: 1 October 2019

Published online: 06 February 2020

\section{References}

1. Haynes RJ, Goh KM. Review on physiological pathways of foliar absorption. Sci Hortic. 1977;7(4):291-302.
2. Taiz L, Zeiger E. Plant physiology. Fifth Edition. Sinauer Associates; 2010.

3. Arand K, Asmus E, Popp C, Schneider D, Riederer M. The mode of action of adjuvants - relevance of physicochemical properties for effects on the foliar application, cuticular permeability and greenhouse performance of Pinoxaden. J Agri Food Chem. 2018;66(23):5770-7.

4. Schönherr J, Baur P. Modelling penetration of plant cuticles by crop protection agents and effects of adjuvants on their rates of penetration. Pestic Sci. 1994;42(3):185-208.

5. Schönherr J, Luber M. Cuticular penetration of potassium salts: effects of humidity, anions, and temperature. Plant Soil. 2001;236(1):117-22.

6. Schönherr J. Cuticular penetration of calcium salts: effects of humidity, anions, and adjuvants. J Plant Nutr Soil Sci. 2001;164(2):225-31.

7. Schönherr J. Characterization of aqueous pores in plant cuticles and permeation of ionic solutes. J Exp Bot. 2006;57(11):2471-91.

8. Tredenick EC, Farrell TW, Forster WA, Psaltis ST. Nonlinear porous diffusion modeling of hydrophilic lonic agrochemicals in astomatous plant cuticle aqueous pores: a mechanistic approach. Front Plant Sci. 2017;10(8):746.

9. Miller JM, Dahan A, Gupta D, Varghese S, Amidon GL. Quasi-equilibrium analysis of the ion-pair mediated membrane transport of low-permeability drugs. J Control Release. 2009;137(1):31-7.

10. Miller JM, Dahan A, Gupta D, Varghese S, Amidon GL. Enabling the intestinal absorption of highly polar antiviral agents: ion-pair facilitated membrane permeation of zanamivir heptyl ester and guanidino oseltamivir. Mol Pharm. 2010;7(4):1223-34.

11. Velický M, Tam KY, Dryfe RA. Permeation of a fully ionized species across a polarized supported liquid membrane. Anal Chem. 2012;84(5):2541-7.

12. Kansy M, Senner F, Gubernator K. Physicochemical high throughput screening: parallel artificial membrane permeation assay in the description of passive absorption processes. J Med Chem. 1998;41(7):1007-10.

13. Hofstetter S, Beck A, Trapp S, Buchholz A. How to design for a tailored subcellular distribution of systemic agrochemicals in plant tissues. J Agri Food Chem. 2018;66(33):8687-97.

14. Yeats TH, Rose JK. The formation and function of plant cuticles. Plant Physiol. 2013;163(1):5-20.

15. Oliveira AF, Meirelles ST, Salatino A. Epicuticular waxes from caatinga and cerrado species and their efficiency against water loss. Anais da Academia Brasileira de Ciencias. 2003;75(4):431-9.

16. White TR, Renzelman CM, Rand AC, Rezai T, McEwen CM, Gelev VM, Turner RA, Linington RG, Leung SS, Kalgutkar AS, Bauman JN. On-resin $\mathrm{N}$-methylation of cyclic peptides for discovery of orally bioavailable scaffolds. Nat Chem Biol. 2011;7(11):810.

17. Nirasay S, Mouget Y, Marcotte I, Claverie JP. Supported bilayer on a nanopatterned membrane as model PAMPA membranes. Int J Pharm. 2011;421(1):170-5.

18. Vigneault B, Percot A, Lafleur M, Campbell PG. Permeability changes in model and phytoplankton membranes in the presence of aquatic humic substances. Environ Sci Technol. 2000;34(18):3907-13.

19. Ojwang'LM, Cook RL. Environmental conditions that influence the ability of humic acids to induce permeability in model biomembranes. Environ Sci Technol. 2013;47(15):8280-7.

20. Schreiber L. Polar paths of diffusion across plant cuticles: new evidence for an old hypothesis. Ann Bot. 2005;95(7):1069-73.

21. Shinoda W. Permeability across lipid membranes. Biochimica et Biophysica Acta (BBA)-Biomembr. 2016;1858(10):2254-65.

\section{Publisher's Note}

Springer Nature remains neutral with regard to jurisdictional claims in published maps and institutional affiliations. 